\title{
Microfluidic technologies for accelerating the clinical translation of nanoparticles
}

\section{Citation}

Valencia, Pedro M., Omid C. Farokhzad, Rohit Karnik, and Robert Langer. 2012. Microfluidic Technologies for Accelerating the Clinical Translation of Nanoparticles. Nature Nanotechnology 7 (10) (October 8): 623-629. doi:10.1038/nnano.2012.168.

\section{Published Version}

doi:10.1038/nnano.2012.168

\section{Permanent link}

http://nrs.harvard.edu/urn-3:HUL.InstRepos:29293166

\section{Terms of Use}

This article was downloaded from Harvard University's DASH repository, and is made available under the terms and conditions applicable to Other Posted Material, as set forth at http:// nrs.harvard.edu/urn-3:HUL.InstRepos:dash.current.terms-of-use\#LAA

\section{Share Your Story}

The Harvard community has made this article openly available.

Please share how this access benefits you. Submit a story.

Accessibility 


\title{
Microfluidic technologies for accelerating the clinical translation of nanoparticles
}

\author{
Pedro M. Valencia ${ }^{1}$, Omid C. Farokhzad $2,3,{ }^{,}$, Rohit Karnik ${ }^{4,}$, , and Robert Langer ${ }^{1,3,{ }^{*}}$ \\ ${ }^{1}$ Department of Chemical Engineering, Massachusetts Institute of Technology, Cambridge, \\ Massachusetts 02139, USA \\ ${ }^{2}$ Laboratory of Nanomedicine and Biomaterials and Department of Anaesthesiology, Brigham and \\ Women's Hospital, Harvard Medical School, Boston, Massachusetts 02115, USA \\ ${ }^{3}$ MIT-Harvard Center for Cancer Nanotechnology Excellence, Massachusetts Institute of \\ Technology, Cambridge, Massachusetts 02139, USA \\ ${ }^{4}$ Department of Mechanical Engineering, Massachusetts Institute of Technology, Cambridge, \\ Massachusetts 02139, USA.
}

\begin{abstract}
Using nanoparticles for therapy and imaging holds tremendous promise for the treatment of major diseases such as cancer. However, their translation into the clinic has been slow because it remains difficult to produce nanoparticles that are consistent 'batch-to-batch', and in sufficient quantities for clinical research. Moreover, platforms for rapid screening of nanoparticles are still lacking. Recent microfluidic technologies can tackle some of these issues, and offer a way to accelerate the clinical translation of nanoparticles. In this Progress Article, we highlight the advances in microfluidic systems that can synthesize libraries of nanoparticles in a well-controlled, reproducible and high-throughput manner. We also discuss the use of microfluidics for rapidly evaluating nanoparticles in vitro under microenvironments that mimic the in vivo conditions. Furthermore, we highlight some systems that can manipulate small organisms, which could be used for evaluating the in vivo toxicity of nanoparticles or for drug screening. We conclude with a critical assessment of the near- and long-term impact of microfluidics in the field of nanomedicine.
\end{abstract}

\begin{abstract}
Nanomedicine is the application of nanotechnology to medicine, specifically involving the use of engineered nanomaterials for therapy and diagnosis of major diseases such as cancer, cardiovascular and infectious diseases ${ }^{1}$. The first generation of nanoparticles with applications in medicine dates back to the 1970s, when drug-loaded nanoscale liposomes were developed to deliver their cargo to diseased cells in a 'Trojan horse' fashion ${ }^{2}$. Since then, a new generation of targeted drug delivery vehicles (for example, polymeric nanoparticles) ${ }^{3}$, contrast agents (such as iron oxide nanoparticles) $)^{4}$, diagnostic tools ${ }^{5}$, and antennas for photothermal therapy (for example, gold nanoparticles) ${ }^{6}$ have emerged. This is driven in part by further understanding of the biology of diseased states, and by
\end{abstract}

\footnotetext{
(C) 2012 Macmillan Publishers Limited. All rights reserved

*Correspondence and requests for materials should be addressed to R.L., R.K. and O.C.F. ofarokhzad@zeus.bwh.harvard.edu; karnik@mit.edu; rlanger@mit.edu.

Additional information The authors declare competing financial interests: O.C.F. and R.L. disclose financial interest in BIND Biosciences and Selecta Biosciences, two biotechnology companies developing nanoparticle technologies for medical applications. BIND and Selecta did not support the aforementioned work, and at present these companies have no rights to any technology or intellectual property developed as part of this work.

Reprints and permissions information is available online at www.nature.com/reprints.
} 
technological advances in imaging techniques and synthesis of novel biocompatible and biodegradable materials ${ }^{7}$. Now, nanomedicine promises the precise delivery of drugs to disease sites (such as tumours and atherosclerotic plaques) without off-target toxicities, and the early detection of diseases using selective contrast agents and sensitive diagnostic tools ${ }^{8}$.

Nanoparticles are attractive in medicine because their surfaces can be chemically modified for targeting specific disease tissues, or for in vivo stability. For therapy, drugs can be encapsulated inside nanoparticles and released in a controlled manner over time. For imaging, nanoparticles can provide higher contrast (for example, iron oxide nanoparticles for magnetic resonance imaging) or higher brightness (for example, quantum dots (QDs) for fluorescence imaging) than conventional small-molecule agents ${ }^{9}$. Despite these advantages and several decades of research, only a handful of nanoparticles have received approval from the US Food and Drug Administration. Examples include iron oxide nanoparticles for magnetic resonance imaging (for example, Feridex and Resovist), liposomes encapsulating the anticancer drug, doxorubicin, for chemotherapy (known as Doxil), and the protein-based nanoparticle encapsulating paclitaxel for chemotherapy (called Abraxane) ${ }^{10}$.

In fact, translation of nanoparticles to the clinic has been slow compared with smallmolecule drugs, with the majority of nanoparticles not even reaching the point of in vivo evaluation, and even fewer reaching clinical trials. This is due to a combination of factors. It remains difficult to reproducibly synthesize batches of nanoparticles that have identical properties and in sufficient quantities for clinical applications ${ }^{11}$. Moreover, knowledge on the fate of nanoparticles at the body-, organ- and cell-level remains limited ${ }^{12}$; this makes rational design of nanoparticles difficult and necessitates the use of screening-based approaches for synthesis. Furthermore, there are few platforms that can rapidly evaluate the biological behaviour of nanoparticles in vitro under conditions that can be correlated with their performance in vivo ${ }^{11}$. For example, there is a need for high-throughput methods for evaluating the binding and internalization of nanoparticles by cells, or the interaction of nanoparticles with plasma proteins and the complement system, among others. Finally, there is insufficient understanding of the biophysical and chemical interactions of nanoparticles with proteins, membranes, DNA and organelles. These interactions could have either beneficial or adverse outcomes ${ }^{13}$. It is expected that technologies tackling some of these challenges could significantly accelerate the discovery and clinical translation of nanomedicines.

Microfluidics - the science and technology of manipulating nanolitre volumes in microscale fluidic channels - has impacted a range of applications, including biological analysis, chemical synthesis, single-cell analysis and tissue engineering ${ }^{14}$. Building on its origins in semiconductor technology and chemical separations, the expansion of microfluidics has been driven by its ability to process small sample volumes and access biologically relevant length scales and microscale transport phenomena. This expansion has been largely facilitated by techniques, such as soft lithography, that enable rapid design and prototyping of microfluidic devices using a variety of materials ${ }^{14}$. Recent advances and innovations in microfluidics are expected to improve the synthesis of nanoparticles and accelerate their transition to clinical evaluation (Fig. 1). Although many of these microfluidic systems are still being developed, they have the potential to become widely adopted because they are economical, reproducible, amenable to modifications and can be integrated with other technologies ${ }^{15}$. In this Progress Article, we highlight some of these technologies and discuss their impact on accelerating the clinical translation of nanoparticles. 


\section{Well-controlled synthesis of nanoparticles}

Amphiphilic molecules such as block copolymers and lipids can self-assemble into nanoparticles when they experience a change in solvent quality (for example, from organic solvent to aqueous) (Fig. 2a). A common and flexible way to accomplish a change in solvent quality is by mixing the solvent with the anti-solvent, where mixing time directly influences the final size and size distribution of the nanoparticles formed ${ }^{16}$. If the mixing timescale, $\tau_{\text {mix }}$, is longer than the characteristic timescale for chains to nucleate and grow ( $\tau_{\text {agg }}, \sim 10-$ $100 \mathrm{~ms}$ depending on the molecular weight of the chain), the nanoparticles begin to assemble under varying degrees of solvent quality. This heterogeneous environment prevents effective stabilization of the nanoparticles by the hydrophilic portion of the amphiphilic molecule and facilitates their aggregation, leading to the formation of larger, polydisperse nanoparticles. However, if $\tau_{\text {mix }}<\tau_{\text {agg }}$, particle self-assembly occurs primarily when the solvent change is complete. This homogenous solvent environment for nanoparticle assembly allows the hydrophilic portion of the molecule to stabilize the nanoparticles more effectively, and this yields smaller nanoparticles with uniform size ${ }^{17}$. Although conventional bulk mixing occurs at the timescale of seconds, in microfluidic devices the mixing time of solvents is controllable and tunable from the millisecond to microsecond scale (reaching $\left.\tau_{\text {mix }}<\tau_{\text {agg }}\right)^{16,18}$.

In recent years, several microfluidic systems that enable rapid mixing without the need of external actuators, such as stirrers or electric fields, have been developed ${ }^{19}$. The most widely used include flow-focusing mixers ${ }^{20}$, droplet mixers ${ }^{21}$ and those with micromixing structures embedded inside the channel ${ }^{22}$. Flow focusing squeezes the solvent stream between two anti-solvent streams, resulting in rapid solvent exchange via diffusion (Fig. 2b). Droplets and three-dimensional microchannel geometries result in complex folding of fluid flows, which can completely mix two or more streams in milliseconds (Fig. 2b). The implementation of these mixing techniques for the formation of organic nanoparticles in continuous flow has resulted in polymeric and lipid nanoparticles with tunable nanoparticle size, narrower size distribution, higher drug loadings and greater batch-to-batch reproducibility relative to those made with conventional bulk techniques ${ }^{23}$ (Fig. 2c).

Similarly, inorganic nanoparticles comprising transition metals such as gold, iron and cadmium, among others, undergo self-assembly where metal solutes nucleate, grow and agglomerate into nanoclusters (Fig. 2d) ${ }^{24}$. Obtaining narrow particle-size distribution requires rapid nucleation followed by growth of nanoparticles to the desired size in the absence of further nucleation, which can be accomplished by controlling the mixing time of reagents, reaction temperature and reaction time ${ }^{25}$. In bulk, these parameters are difficult to control, leading to uneven mixing, local temperature fluctuations and uncontrolled reaction times $^{25}$. In contrast, microfluidic devices allow for control over the mixing time by varying solvent flow rates or channel geometry. Moreover, better heat transfer owing to large surface areas enables better temperature control, preventing the formation of large temperature gradients. Finally, as the channel length directly corresponds to the time taken by the reactants to flow through it in continuous flow synthesis, the reaction time can be controlled by tuning the channel length or by adding reagents at precise downstream locations during the particle formation process to quench the reaction ${ }^{26}$.

Two-phase droplet mixers where reagents are encapsulated in droplets and separated by inert fluids are commonly used for the synthesis of inorganic nanoparticles (Fig. 2e) ${ }^{27}$. In this configuration, rapid mixing of the solutions occurs inside the droplets, which serve as identical microscale reactors providing homogeneous conditions for nanoparticle nucleation and growth. Both droplet-based and single-phase systems have been used to synthesize QDs that exhibit narrow size distributions, which translates into sharper absorption peaks and 
better luminescence qualities ${ }^{27}$, with control over size to tune the absorption spectra ${ }^{28}$ (Fig. 2f). Finally, similar systems have been implemented in the controlled synthesis of gold nanoparticles of defined size and shape, iron oxide nanoparticles with higher magnetization, and QDs with controlled size and biocompatible coatings ${ }^{29}$.

Over the past four years, several examples showing the use of microfluidics for the synthesis of nanoparticles with different size, shape and surface compositions have emerged ${ }^{30}$. At present, there are microfluidic systems capable of characterizing the nanoparticle size and stability following synthesis in a single platform ${ }^{31,32}$. Moreover, large numbers of distinct nanoparticles can be obtained through combinatorial synthesis ${ }^{33}$, and production rates of identical nanoparticles can be increased through parallelization or re-design of the devices ${ }^{34,35}$. Similar to high-throughput synthesis of libraries of small molecules, these advantages could potentially enable screening and optimization of libraries of nanoparticles with distinct properties. One of the challenges in gene therapy, for example, is finding the right formulation for delivering nucleic acids to specific sites in the body. By mixing different precursors at varying ratios, microfluidic systems have enabled a one-step combinatorial synthesis of libraries of polymeric and lipid nanoparticles that encapsulate DNA and small-interfering RNA, respectively. Screening these libraries of nanoparticles has helped identify superior formulations for gene transfection, compared with conventional transfection agents such as lipofectamine 2000. Furthermore, using this method, potent lipid-based small-interfering RNA formulations for in vivo delivery to the liver have also been discovered ${ }^{33,34}$.

\section{Evaluation and screening of nanoparticles}

Another challenge in nanoparticle development is the lack of in vitro models capable of predicting in vivo behaviour ${ }^{36}$. Conventionally, nanoparticles are evaluated in vitro using cells cultured in well plates, which does not capture the complexity of nanoparticle-cell interactions in vivo. For instance, a recent study showed that sedimentation of gold nanoparticles in well plates could lead to misinterpretation of results, such as increased nanoparticle uptake ${ }^{37}$. Microfluidics provides significant advantages over conventional methods for cell and tissue culture by displaying structures and networks at relevant physiological length scales, and by incorporating fluid flow and mechanical forces that bring the cell-based assays a step closer to mimicking the in vivo microenvironment ${ }^{38}$ (Fig. 3a). This is especially advantageous, for instance, when investigating the toxic effects related to the cell uptake of nanoparticles. A recently developed microfluidic system for evaluating QD toxicity on mouse fibroblasts revealed increased cell viability under flow conditions compared with static incubation, possibly due to the absence of QD sedimentation ${ }^{39}$.

Recently, researchers have focused on developing biomimetic microfluidic technologies capable of portraying organ-level functions on a chip, such as those observed in the lung, liver and kidneys, among others ${ }^{38,40}$. For instance, microfluidic systems that reconstitute the critical functional alveolar-capillary liquid/air interface of the human lung have been recently fabricated by growing alveolar epithelial cells and microvascular endothelial cells on different sides of a perforated silicone membrane. The membrane was pneumatically actuated to mimic the physiological expansion-contraction motion due to breathing. It was found that cyclic mechanical strain accentuates toxic and inflammatory responses in the lung when exposed to silica nanoparticles, which could not have been observed with other conventional in vitro systems ${ }^{41}$ (Fig. 3b). Using a similar design approach, a 'gut-on-a-chip' was developed by coating both sides of a membrane separating two microfluidic devices with extracellular matrix and lined by human intestinal epithelial cells. It was demonstrated that by subjecting the membrane to flow and cyclic strains similar to those encountered in 
the gut, villi-like structures were formed and the co-culture of the intestinal microbes was made possible 42 (Fig. 3c).

Expansion of these technologies to other organs, for instance 'liver-on-a-chip"43, could lead to platforms for evaluating and screening nanoparticle toxicity in organs where nanoparticles tend to accumulate and toxicity is likely to be a major concern (for example, liver, spleen and kidney). Although nanoparticles would still need to be evaluated in animals, such microfluidic systems could take in vitro nanoparticle screening to a new level of utility by selecting promising candidates with higher probabilities of success from a large pool of nanoparticle formulations, and eliminating those that would otherwise have failed in larger animal studies. Furthermore, coupling these technologies with microfluidic devices used for nanoparticle synthesis opens the possibility of rapid combinatorial screening of a large number of different nanoparticles under various conditions (for example, concentrations, $\mathrm{pH}$ values, under the presence of specific proteins and so on).

Nanoparticles exhibiting promising results in vitro are subsequently evaluated in vivo, which is considerably more expensive and resource intensive, especially in non-human primates. Although most of the parameters, such as pharmacokinetics, biodistribution and efficacy, are evaluated in mice and larger animals, tracking physiological effects of nanoparticles on animal development could potentially be obtained using a large number of smaller organisms. The zebrafish and Caenorhabditis elegans worms are well-known models for studying fundamental mechanisms and progression of human diseases, and for drug screening ${ }^{44}$. For example, the zebrafish was recently used as an in vivo model to develop a hazard ranking for engineered nanoparticles based on their impact on mortality rate and morphological defects in zebrafish embryos exposed to these materials ${ }^{45}$. However, current methods for manipulating these organisms generally suffer from low throughput, low automation and imprecise delivery of external stimuli ${ }^{46}$. To solve these challenges, engineered microfluidic systems with dimensions comparable to small organisms and containing valves and suction points have been developed. These systems enable precise manipulation of these organisms with respect to placement and orientation for highthroughput screening 46,47 (Fig. 3d). Other microfluidic systems are being developed that are capable of imaging dynamic cellular processes in small organisms, such as cell division and migration, degeneration, aging and regeneration ${ }^{48}$. With such technologies in place, it might be possible to use real-time microscopy to track physiological responses to fluorescently labelled nanotherapeutics and nano-imaging agents, as well as assess the distribution and efficacy of nanoparticles at both the organ and body level. Furthermore, real-time tracking of nanoparticle-induced toxicity at different concentrations and conditions in small organisms could enable rapid selection of nanoparticles (especially those made with novel synthetic materials) that are more likely to be non-toxic in larger animals.

\section{Future prospects}

At present, the field of microfluidics applied to nanomedicine is still in its infancy. Although nanoparticles have a relatively small footprint in the pharmaceutical industry, it is anticipated that as these products bring in revenue, industry-led research and development efforts would probably adopt technologies, such as microfluidics, to accelerate their development. Nevertheless, microfluidic technologies, such as organ-on-a-chip and smallanimal screening, are likely to be adopted first for the screening of small-molecule drug candidates, where the need for such tools is evident.

There are a few key directions at the intersection of microfluidics and nanomedicine that are likely to be pursued in the near future (Table 1). Although the quantity of nanoparticles synthesized by microfluidic devices is often in the micro- to milligram range, parallel and 
stackable microfluidic systems could continuously produce nanoparticles on the gram to kilogram scale with the same properties as those prepared at the bench scale. Similarly, the use of microfluidic platform technologies to reproducibly synthesize and screen libraries of nanoparticles with different chemical compositions and/or physical and chemical properties could potentially advance nanoparticle discovery analogously to how the high-throughput screening of small molecules in medicinal chemistry advanced small-molecule discovery. With respect to the design and development of novel nanoparticle constructs, the use of microfluidics could enable the synthesis of nanoparticles with properties not accessible by conventional synthesis, similar to what has already occurred for microparticle synthesis ${ }^{49}$.

Another avenue of future research will be the integration of different steps of nanoparticle development into a single system (for example, nanoparticle synthesis characterization and evaluation), together with feedback control through a combination of microfluidics, robotics and automation, thus significantly cutting the time and cost of nanoparticle development. Finally, mass-produced microfluidic devices and well-defined nanoparticle precursors can aid in the synthesis of identical batches of nanoparticles with little to no variations introduced by user handling. This could lead to the use and commercialization of 'nanoparticle synthesis kits' composed of calibrated devices that can reproducibly synthesize a specific class of nanoparticle with well-defined properties for use as standards in conventional toxicological assays. Considering the large number of nanoparticles being made of novel synthetic materials or of unusual shapes, such standardization would be highly useful for regulatory purposes, among others.

Microfluidic technologies are capable of accelerating the discovery and translation of nanoparticles, and could serve as a tool for nanotherapeutics to reach a similar 'tipping point' reached by genome sequencing in the past decade after high-throughput sequencing technologies were developed ${ }^{50}$. Among all the microfluidic technologies, those developed for synthesis and in vitro screening of nanoparticles have the highest probability of making an impact in the near future (Table 1). Specifically, microfluidic synthesis may be adopted as a second-generation manufacturing technology after the initial success of US Food and Drug Administration-approved nanoparticles in cases where the advantages of microfluidic synthesis are significant. Microfluidic synthesis may also be adopted as a screening tool to identify optimal nanoparticles in academic and industrial research laboratories.

Alternatively, the impact of microfluidics might be observable in the medium- to long-term future for nanoparticle characterization and in vivo evaluation. For nanoparticle characterization, the use of microfluidics would probably increase once more-advanced technologies are developed to characterize several nanoparticle properties (for example, size, charge, surface composition and stability) in a single system. Similarly, in vivo evaluation of nanoparticles in microfluidics would probably mature once both easily adoptable microfluidic systems for manipulating small organisms and methods for translating data obtained from these organisms to larger animals are developed.

Overall, the use of microfluidic technologies in nanomedicine brings exciting opportunities to expand the body of knowledge in the field, advance the clinical translation of nano-based therapeutics and imaging agents, and demonstrate innovative ways to develop other classes of drugs.

\section{Acknowledgments}

This work was supported by the Koch-Prostate Cancer Foundation Award in Nanotherapeutics (R.L. and O.C.F.), the National Cancer Institute Center of Cancer Nanotechnology Excellence at MIT-Harvard (U54-CA151884, R.L. and O.C.F.), and the National Heart, Lung, and Blood Institute Programs of Excellence in Nanotechnology (HHSN268201000045C; R.L. and O.C.F.). P.M.V. is supported by the National Science Foundation graduate 
research fellowship. We thank B. Timko and F. Karim for assistance in drafting Figs 1 and 2, respectively. We also thank A. Radovic-Moreno, C. Alabi and E. Pridgen for their insightful comments.

\section{References}

1. Petros RA, DeSimone JM. Strategies in the design of nanoparticles for therapeutic applications. Nature Rev. Drug. Discov. 2010; 9:615-627. [PubMed: 20616808]

2. Gregoriadis G. Drug entrapment in liposomes. FEBS Lett. 1973; 36:292-296. [PubMed: 4763309]

3. Hrkach J, et al. Preclinical development and clinical translation of a PSMA-targeted docetaxel nanoparticle with a differentiated pharmacological profile. Sci. Transl. Med. 2012; 4:128ra39. This article describes the translation of the first targeted polymeric nanoparticle for drug delivery from discovery to clinical trials.

4. Qiao R, Yang C, Gao M. Superparamagnetic iron oxide nanoparticles: from preparations to in vivo MRI applications. J. Mater. Chem. 2009; 19:6274-6293.

5. Haun JB, et al. Micro-NMR for rapid molecular analysis of human tumor samples. Sci. Transl. Med. 2011; 3:71ra16.

6. Kim BY, Rutka JT, Chan WC. Nanomedicine. N. Engl. J. Med. 2010; 363:2434-2443. [PubMed: 21158659]

7. Kamaly N, Xiao Z, Valencia PM, Radovic-Moreno AF, Farokhzad OC. Targeted polymeric therapeutic nanoparticles: design, development and clinical translation. Chem. Soc. Rev. 2012; 41:2971-3010. [PubMed: 22388185]

8. Peer D, et al. Nanocarriers as an emerging platform for cancer therapy. Nature Nanotech. 2007; 2:751-760.

9. Barreto JA, et al. Nanomaterials: applications in cancer imaging and therapy. Adv. Mater. 2011; 23:H18-H40. [PubMed: 21433100]

10. Shi J, Xiao Z, Kamaly N, Farokhzad OC. Self-assembled targeted nanoparticles: evolution of technologies and bench to bedside translation. Acc. Chem. Res. 2011; 44:1123-1134. [PubMed: 21692448]

11. Murday JS, Siegel RW, Stein J, Wright JF. Translational nanomedicine: status assessment and opportunities. Nanomedicine. 2009; 5:251-273. [PubMed: 19540359]

12. Chou LY, Ming K, Chan WC. Strategies for the intracellular delivery of nanoparticles. Chem. Soc. Rev. 2011; 40:233-245. [PubMed: 20886124]

13. Nel AE, et al. Understanding biophysicochemical interactions at the nano-bio interface. Nature Mater. 2009; 8:543-557. [PubMed: 19525947]

14. Whitesides GM. The origins and the future of microfluidics. Nature. 2006; 442:368-373. [PubMed: 16871203] An excellent classic review on the present and future of microfluidics by one of the fathers of the field, George Whitesides.

15. DeMello AJ. Control and detection of chemical reactions in microfluidic systems. Nature. 2006; 442:394-402. [PubMed: 16871207]

16. Johnson BK, Prud'homme RK. Mechanism for rapid self-assembly of block copolymer nanoparticles. Phys. Rev. Lett. 2003; 91:118302. [PubMed: 14525460] This article describes the mechanism of nanoparticle self-assembly and explains how rapid mixing is key in controlling nanoparticle size.

17. Chen T, Hynninen AP, Prud'homme RK, Kevrekidis IG, Panagiotopoulos AZ. Coarse-grained simulations of rapid assembly kinetics for polystyrene- $b$-poly(ethylene oxide) copolymers in aqueous solutions. J. Phys. Chem. B. 2008; 112:16357-16366. [PubMed: 19367859]

18. Karnik R, et al. Microfluidic platform for controlled synthesis of polymeric nanoparticles. Nano Lett. 2008; 8:2906-2912. [PubMed: 18656990]

19. Capretto L, Cheng W, Hill M, Zhang X. Micromixing within microfluidic devices. Top. Curr. Chem. 2011; 304:27-68. [PubMed: 21526435]

20. Rhee M, et al. Synthesis of size-tunable polymeric nanoparticles enabled by 3D hydrodynamic flow focusing in single-layer microchannels. Adv. Mater. 2011; 23:H79-H83. [PubMed: 21433105] 
21. Liu K, et al. A digital microfluidic droplet generator produces self-assembled supramolecular nanoparticles for targeted cell imaging. Nanotechnology. 2010; 21:445603. [PubMed: 20935351]

22. Valencia PM, et al. Single-step assembly of homogenous lipid-polymeric and lipid-quantum dot nanoparticles enabled by microfluidic rapid mixing. ACS Nano. 2010; 4:1671-1679. [PubMed: 20166699]

23. Jahn A, et al. Preparation of nanoparticles by continuous-flow microfluidics. J. Nanopart. Res. 2008; 10:925-934.

24. Besson C, Finney EE, Finke RG. A mechanism for transition-metal nanoparticle self-assembly. J. Am. Chem. Soc. 2005; 127:8179-8184. [PubMed: 15926847]

25. Song Y, Hormes J, Kumar CS. Microfluidic synthesis of nanomaterials. Small. 2008; 4:698-711. [PubMed: 18535993]

26. Gu FX, et al. Targeted nanoparticles for cancer therapy. Nano Today. 2007; 2:14-21.

27. Shestopalov I, Tice JD, Ismagilov RF. Multi-step synthesis of nanoparticles performed on millisecond time scale in a microfluidic droplet-based system. Lab Chip. 2004; 4:316-321. [PubMed: 15269797]

28. Kikkeri R, Laurino P, Odedra A, Seeberger PH. Synthesis of carbohydrate-functionalized quantum dots in microreactors. Angew. Chem. Int. Ed. 2010; 49:2054-2057.

29. Marre S, Jensen KF. Synthesis of micro and nanostructures in microfluidic systems. Chem. Soc. Rev. 2010; 39:1183-1202. [PubMed: 20179831]

30. Zhao CX, He LZ, Qiao SZ, Middelberg APJ. Nanoparticle synthesis in microreactors. Chem. Eng. Sci. 2011; 66:1463-1479.

31. Fraikin JL, Teesalu T, McKenney CM, Ruoslahti E, Cleland AN. A high-throughput label-free nanoparticle analyser. Nature Nanotech. 2011; 6:308-313.

32. Birnbaumer G, et al. Rapid liposome quality assessment using a lab-on-a-chip. Lab Chip. 2011; 11:2753-2762. [PubMed: 21691661]

33. Wang $\mathrm{H}$, et al. A rapid pathway toward a superb gene delivery system: programming structural and functional diversity into a supramolecular nanoparticle library. ACS Nano. 2010; 4:6235-6243. [PubMed: 20925389] This article is one of the first examples that exploit microfluidic systems for rapid combinatorial synthesis of nanoparticles with a variety of physical and chemical properties.

34. Chen D, et al. Rapid discovery of potent siRNA-lipid-nanoparticles enabled by controlled microfluidic formulation. J. Am. Chem. Soc. 2012; 134:6948-6951. [PubMed: 22475086]

35. Kim Y, et al. Mass production and size control of lipid-polymer hybrid nanoparticles through controlled microvortices. Nano Lett. 2012; 12:3587-3591. [PubMed: 22716029]

36. Dobrovolskaia MA, Germolec DR, Weaver JL. Evaluation of nanoparticle immunotoxicity. Nature Nanotech. 2009; 4:411-414.

37. Cho EC, Zhang Q, Xia Y. The effect of sedimentation and diffusion on cellular uptake of gold nanoparticles. Nature Nanotech. 2011; 6:385-391.

38. Ziolkowska K, Kwapiszewski R, Brzozka Z. Microfluidic devices as tools for mimicking the in vivo environment. New J. Chem. 2011; 35:979-990.

39. Mahto SK, Yoon TH, Rhee SW. A new perspective on in vitro assessment method for evaluating quantum dot toxicity by using microfluidics technology. Biomicrofluidics. 2010; 4:034111. [PubMed: 20957065]

40. Huh D, Torisawa YS, Hamilton GA, Kim HJ, Ingber DE. Microengineered physiological biomimicry: organs-on-chips. Lab Chip. 2012; 12:2156-2164. [PubMed: 22555377]

41. Huh D, et al. Reconstituting organ-level lung functions on a chip. Science. 2010; 328:1662-1668. [PubMed: 20576885] This article describes the design and assembly of a microfluidic system that recreates the alveolar-endothelial interface in lungs.

42. Kim HJ, Huh D, Hamilton G, Ingber DE. Human gut-on-a-chip inhabited by microbial flora that experiences intestinal peristalsis-like motions and flow. Lab Chip. 2012; 12:2165-2174. [PubMed: 22434367]

43. Toh YC, et al. A microfluidic 3D hepatocyte chip for drug toxicity testing. Lab Chip. 2009; 9:2026-2035. [PubMed: 19568671] 
44. Crane MM, Chung K, Stirman J, Lu H. Microfluidics-enabled phenotyping, imaging, and screening of multicellular organisms. Lab Chip. 2010; 10:1509-1517. [PubMed: 20383347]

45. George S, et al. Use of a high-throughput screening approach coupled with in vivo zebrafish embryo screening to develop hazard ranking for engineered nanomaterials. ACS Nano. 2011; 5:1805-1817. [PubMed: 21323332]

46. Shi W, Wen H, Lin B, Qin J. Microfluidic platform for the study of Caenorhabditis elegans. Top. Curr. Chem. 2011; 304:323-338. [PubMed: 21516386]

47. Baker M. Screening: the age of fishes. Nature Meth. 2011; 8:47-51.

48. Samara C, et al. Large-scale in vivo femtosecond laser neurosurgery screen reveals small-molecule enhancer of regeneration. Proc. Natl Acad. Sci. USA. 2010; 107:18342-18347. [PubMed: 20937901]

49. Dendukuri D, Doyle PS. The synthesis and assembly of polymeric microparticles using microfluidics. Adv. Mater. 2009; 21:4071-4086.

50. Zhao J, Grant SF. Advances in whole genome sequencing technology. Curr. Pharm. Biotechnol. 2011; 12:293-305. [PubMed: 21050163] 


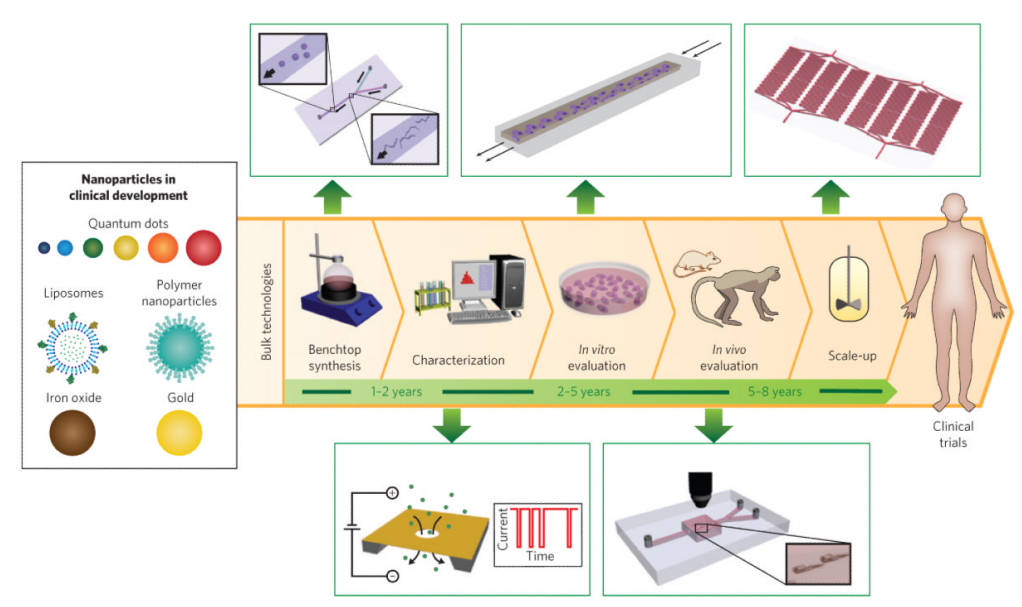

Figure 1. Nanoparticles in clinical development, steps for their translation (with average timescales) and microfluidic methods (green boxes) that could improve or complement current technologies

Synthesis is carried out in large reaction flasks, whereas microfluidic synthesis is carried out at micro and nano length scales that allow for improved control over reaction conditions.

Characterization often involves taking a small sample of nanoparticles and measuring their properties offline, whereas nanopores embedded in microfluidic devices allow for real-time, in-line characterization. In vitro evaluation in plate wells produces a microenvironment far from that in vivo, whereas continuous flow in microfluidic systems result in conditions closer to those in vivo. In vivo evaluation in large animals is helpful for estimating the pharmacology of nanoparticles. To complement these studies microfluidic systems could enable real-time tracking of nanoparticles in large numbers of small organisms. Scale-up is generally carried out in reactor vessels several times larger than benchtop flasks, whereas parallelization of microfluidic channels can increase the production rate of nanoparticles with properties identical to the one at bench scale. 


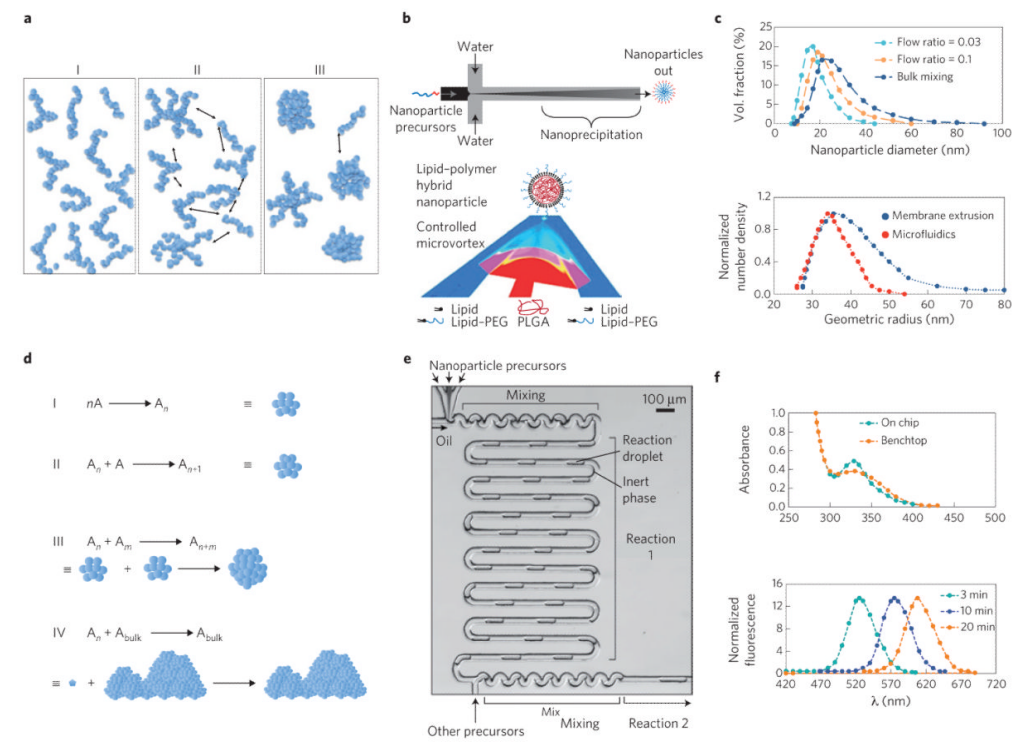

Figure 2. Microfluidic synthesis of nanoparticles

a, Schematic of the self-assembly mechanism of organic nanoparticles. On mixing with antisolvent, polymers (or lipids) are brought to the vicinity of each other (I) then nucleate (II), subsequently aggregating into nanoparticles (III). b, Schematic of microfluidic synthesis of organic nanoparticles by rapid mixing through hydrodynamic flow focusing (top) and microvortices (bottom). Red and dark blue indicate organic and aqueous streams, respectively, while pink and light blue indicate their degree of mixing. PEG, polyethylene glycol; PLGA, poly(lactic-co-glycolic acid). c, Size distribution of polymeric nanoparticles (top) and liposomes (bottom) prepared in microfluidics compared with bulk synthesis. In both cases, narrower particle-size distributions are produced through microfluidics. d, Schematic of the self-assembly mechanism of inorganic nanoparticles. Individual molecules first nucleate (I and II), followed by aggregation of nuclei into nanoparticles (III). If the reaction is not quenched or stabilized, nanoparticles tend to agglomerate into bulk material (IV). A refers to individual molecules forming the nanoparticle, and $\mathrm{A}_{n}$ and $\mathrm{A}_{m}$ refer to nuclei formed of $n$ and $m$ number of A molecules, respectively. e, Microfluidic synthesis of inorganic nanoparticles by rapid mixing through two-phase flow where reagents are embedded in fluid droplets carried by an inert fluid. f, Top: sharp versus broad absorption maximum of QDs synthesized in microchannels and bulk, respectively. Bottom: control of the absorption spectra of QDs as function of reaction time. Figure reproduced with permission from: a, ref. 16, () 2003 APS; b, Top: ref. 18, ( 2008 ACS; Bottom: ref. 35, ( 2012 ACS; c, Top: ref. 18, ( 2008 ACS; Bottom: ref. 23, (C 2008 Springer; d, ref. 24, () 2005 ACS; e, ref. 27, @ 2004 RSC; f, Top: ref. 28, @ 2010 Wiley; Bottom: ref. 27, @ 2004 RSC. 

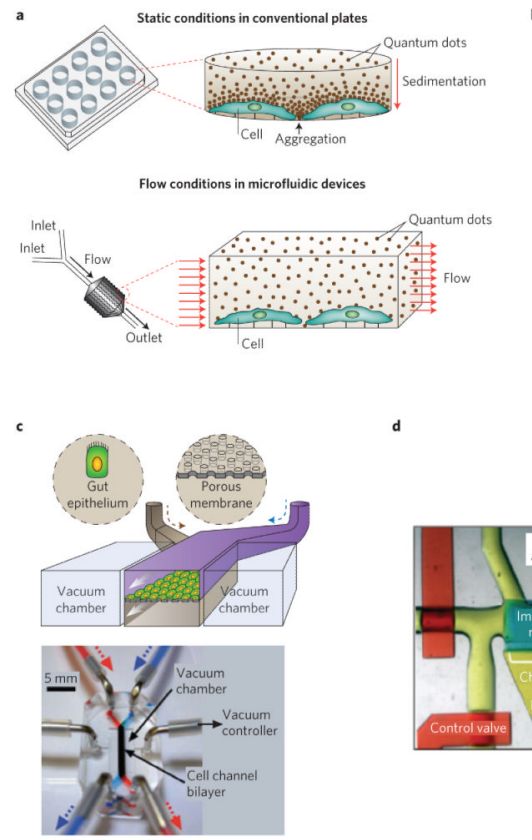
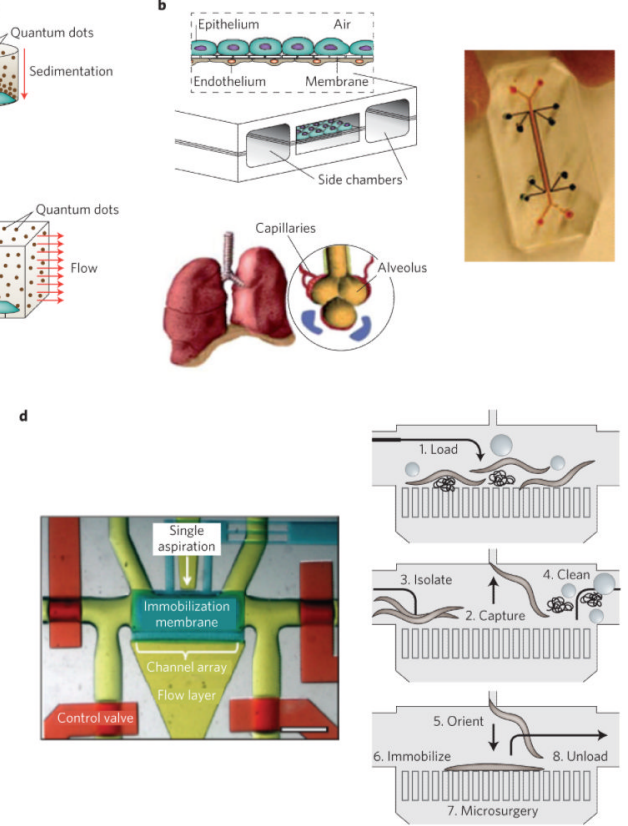

Figure 3. Microfluidic systems for in vitro evaluation and screening of nanoparticles a, Schematic of nanoparticle sedimentation in conventional plates, which could result in misinterpretation of results. In contrast, flow conditions in microfluidics provide a moreaccurate method for evaluating nanoparticles in vitro. b, Left: schematic of the lung-on-achip that reconstitutes the critical functional alveolar-capillary interface of the human lung through a stretchable membrane containing an epithelium layer on one side and an endothelium layer on the other. Right: photograph of actual device. c, Top: schematic of the gut-on-a-chip made by flexible, porous, extracellular matrix-coated membrane lined by gut epithelial cells. The blue and brown arrows indicate two different streams of culture medium separated by a membrane, entering the channel from the top and bottom, respectively. Bottom: photograph of the gut-on-a-chip device made of polydimethylsiloxane elastomer. A syringe pump was used to perfuse dyes (red and blue) for channel visualization. d, Left: photograph of a dye-filled microfluidic system designed to handle $C$. elegans worms. Red, control valve layer; yellow, flow layer; blue, immobilization layer. Scale bar, $1 \mathrm{~mm}$. Right: schematic showing load, capture, orient, immobilization and unload of the worm. Figure reproduced with permission from: a, ref. 39, (C) 2010 AIP; b, ref. 41, (C) 2010 AAAS; c, ref. 42, (C) 2012 RSC; d, ref. 48, (C) 2010 NAS. 
Table 1

Advantages, disadvantages/challenges, stage of development and potential impact of microfluidic systems on different steps in the clinical translation of nanoparticles.

\begin{tabular}{|c|c|c|c|c|}
\hline & antages & Disadvantages/challenges & Stage of development & Potential impact \\
\hline Synthesis & $\begin{array}{ll}\text { - } & \begin{array}{l}\text { Tunable nanoparticle } \\
\text { size }\end{array} \\
\text { - } & \begin{array}{l}\text { Narrower size } \\
\text { distribution }\end{array} \\
\text { - } \quad \text { Reproducible synthesis } \\
\text { - } \quad \begin{array}{l}\text { Potential for high- } \\
\text { throughput synthesis } \\
\text { and optimization of } \\
\text { nanoparticles }\end{array}\end{array}$ & $\begin{array}{l}\text { Solvent and high- } \\
\text { temperature } \\
\text { incompatibility for } \\
\text { low-cost } \\
\text { polydimethylsiloxane } \\
\text { microchannels } \\
\text { Higher costs and } \\
\text { complexities in the } \\
\text { fabrication of glass } \\
\text { and silicon } \\
\text { microdevices }\end{array}$ & $* * * * *$ & $\begin{array}{l}\text { Rapid } \\
\text { combinatorial, } \\
\text { controlled and } \\
\text { reproducible } \\
\text { synthesis of } \\
\text { libraries of } \\
\text { distinct } \\
\text { nanoparticles for } \\
\text { a } \\
\text { specific } \\
\text { application, and/ } \\
\text { or } \\
\text { reference } \\
\text { nanoparticles for } \\
\text { toxicology } \\
\text { studies }\end{array}$ \\
\hline Characterization & $\begin{array}{l}\text { - } \quad \begin{array}{l}\text { Label-free } \\
\text { characterization }\end{array} \\
\text { - } \quad \begin{array}{l}\text { Potential for feedback } \\
\text { control and real-time } \\
\text { nanoparticle } \\
\text { optimization }\end{array}\end{array}$ & $\begin{array}{l}\text { Current methods are } \\
\text { not applicable to all } \\
\text { classes of } \\
\text { nanoparticles } \\
\text { Not all properties can } \\
\text { be characterized, } \\
\text { such as drug } \\
\text { encapsulation and } \\
\text { release, and signal- } \\
\text { to-noise ratio }\end{array}$ & * & $\begin{array}{l}\text { In-line rapid } \\
\text { characterization } \\
\text { and optimization } \\
\text { of } \\
\text { nanoparticles }\end{array}$ \\
\hline In vitro & $\begin{array}{l}\text { Biological conditions } \\
\text { closer to in vivo } \\
\text { microenvironments } \\
\text { Potential for high- } \\
\text { throughout screening of } \\
\text { a large number of } \\
\text { nanoparticles at } \\
\text { different concentrations }\end{array}$ & $\begin{array}{l}\text { Higher costs and } \\
\text { complexities in the } \\
\text { fabrication and } \\
\text { operation compared } \\
\text { with well plates } \\
\text { Might not be } \\
\text { reusable and if } \\
\text { reusable, it would be } \\
\text { difficult to keep } \\
\text { sterile }\end{array}$ & $* * * *$ & $\begin{array}{l}\text { High-throughput } \\
\text { studies of } \\
\text { nanoparticle } \\
\text { toxicity, efficacy, } \\
\text { tumour } \\
\text { penetration and } \\
\text { organ } \\
\text { distribution, } \\
\text { using 'organ-on- } \\
\text { a-chip' systems }\end{array}$ \\
\hline In vivo & $\begin{array}{l}\text { Large number of } \\
\text { organisms could be } \\
\text { used for a single } \\
\text { measurement }\end{array}$ & $\begin{array}{l}\text { Lack of methods to } \\
\text { translate data from } \\
\text { small-scale } \\
\text { organisms to other } \\
\text { species } \\
\text { Pharmacokinetics or } \\
\text { biodistribution } \\
\text { cannot be determined }\end{array}$ & $* *$ & $\begin{array}{l}\text { Real-time } \\
\text { tracking of the } \\
\text { distribution or } \\
\text { toxicity of } \\
\text { nanoparticles on } \\
\text { small-scale } \\
\text { organisms }\end{array}$ \\
\hline Large-scale synthesis & $\begin{array}{l}\text { - } \\
\text { Bentinuous synthesis } \\
\text { - } \quad \begin{array}{l}\text { Pcale reproducibility } \\
\text { Parallelization allows } \\
\text { for tuning scale of } \\
\text { production }\end{array}\end{array}$ & $\begin{array}{l}\text { Difficult to build } \\
\text { systems at low-cost } \\
\text { that are comparable } \\
\text { to a batch reactor } \\
\text { able to prepare grams } \\
\text { or kilograms of } \\
\text { nanoparticles }\end{array}$ & $* * *$ & $\begin{array}{l}\text { Synthesis of } \\
\text { nanoparticles for } \\
\text { human } \\
\text { administration } \\
\text { using } \\
\text { stackable parallel } \\
\text { microfluidic } \\
\text { units }\end{array}$ \\
\hline
\end{tabular}

